\title{
A Survey on Nature-Inspired Medical Image Analysis: A Step Further in Biomedical Data Integration
}

\author{
Leonardo Rundo ${ }^{1,2,3,4}$, Carmelo Militello ${ }^{4}$, Salvatore Vitabile ${ }^{5}$, \\ Giorgio Russo $^{4}$, Evis Sala ${ }^{1,2}$, Maria Carla Gilardi ${ }^{4}$ \\ ${ }^{1}$ Department of Radiology, University of Cambridge, Cambridge, United Kingdom \\ ${ }^{2}$ Cancer Research UK Cambridge Centre, Cambridge, United Kingdom \\ ${ }^{3}$ Department of Informatics, Systems and Communication, University of Milano - Bicocca, \\ Milan, Italy \\ ${ }^{4}$ Institute of Molecular Bioimaging and Physiology, Italian National Research Council, \\ Cefalù (PA), Italy \\ ${ }^{5}$ Department of Biomedicine, Neuroscience and Advanced Diagnostics, University of Palermo, \\ Palermo, Italy
}

\begin{abstract}
Natural phenomena and mechanisms have always intrigued humans, inspiring the design of effective solutions for real-world problems. Indeed, fascinating processes occur in nature, giving rise to an ever-increasing scientific interest. In everyday life, the amount of heterogeneous biomedical data is increasing more and more thanks to the advances in image acquisition modalities and high-throughput technologies. The automated analysis of these large-scale datasets creates new compelling challenges for data-driven and model-based computational methods. The application of intelligent algorithms, which mimic natural phenomena, is emerging as an effective paradigm for tackling complex problems, by considering the unique challenges and opportunities pertaining to biomedical images. Therefore, the principal contribution of computer science research in life sciences concerns the proper combination of diverse and heterogeneous datasets-i.e., medical imaging modalities (considering also radiomics approaches), Electronic Health Record engines, multi-omics studies, and real-time monitoring-to provide a comprehensive clinical knowledge. In this paper, the state-of-the-art of nature-inspired medical image
\end{abstract}

Address for correspondence: Institute of Molecular Bioimaging and Physiology, Italian National Research Council, Contrada Pietra Pollastra-Pisciotto, 90015 Cefalù (PA), Italy. E-mail: mariacarla.gilardi@ibfm.cnr.it 
analysis methods is surveyed, aiming at establishing a common platform for beneficial exchanges among computer scientists and clinicians. In particular, this review focuses on the main natureinspired computational techniques applied to medical image analysis tasks, namely: physical processes, bio-inspired mathematical models, Evolutionary Computation, Swarm Intelligence, and neural computation. These frameworks, tightly coupled with Clinical Decision Support Systems, can be suitably applied to every phase of the clinical workflow. We show that the proper combination of quantitative imaging and healthcare informatics enables an in-depth understanding of molecular processes that can guide towards personalised patient care.

Keywords: Nature-inspired computing, artificial intelligence, medical image analysis, biomedical data integration

\section{Introduction}

Since the dawn of time, nature has always intrigued human lives, serving often as a source of inspiration in the design of effective solutions for real-world problems. Indeed, fascinating processes occur in nature, giving rise to an ever-increasing scientific interest [1]. In real-world applications, many problems are complex or computationally intensive for traditional exhaustive algorithmic methods [2], such as in the case of NP-hard problems [3]. Relying on natural mechanisms, information processing in nature is generally performed by means of distributed and self-organised approaches without requiring a centralised control [4]. These adaptive strategies allow for resiliency and reliability when transferred to computational systems.

Nowadays, the automated analysis of large-scale life science datasets creates new compelling challenges for data-driven and model-based computational methods. As a matter of fact, the amount of heterogeneous biomedical data is increasing more and more thanks to the advances in image acquisition modalities and high-throughput technologies [5, 6]. In addition, electronic health (e-health) [7] and mobile health ( $\mathrm{m}$-health) [8] can be properly integrated to support personalised screening and diagnosis [9]. Therefore, cutting-edge Information and Communication Technology (ICT) can enable the shift from organisation-centric to patient-centric models, leading to collaborative multi-institutional healthcare service delivery processes [10]. Unfortunately, this huge information ensemble could overwhelm the analytic capabilities needed by physicians during their critical decision-making tasks [11]. The application of intelligent algorithms, which mimic natural phenomena, is emerging as an effective paradigm for tackling complex problems, by considering the unique challenges and opportunities pertaining to biomedical images [12]. Indeed, these tasks-which could be difficult to model with constrained-based approaches relying either on dynamic or linear programming — might be stochastic in nature, and can be suitably tackled by global search metaheuristics, allowing for explicitly modeling the uncertainty or the vagueness of natural phenomena [2]. Thus, the abstraction of natural phenomena can enable a fruitful interchange between natural sciences and computer science, leading to the evolution of a novel set of powerful techniques [1]. From a philosophical perspective, thanks to the modelling of emergent behaviours, nature-inspired computing defines a holistic framework as opposed to the reductionist approach of connectionist networks [13]. Therefore, we can argue that working at the interface of computer science and natural sciences may help to unveil the interconnections between these disciplines. 


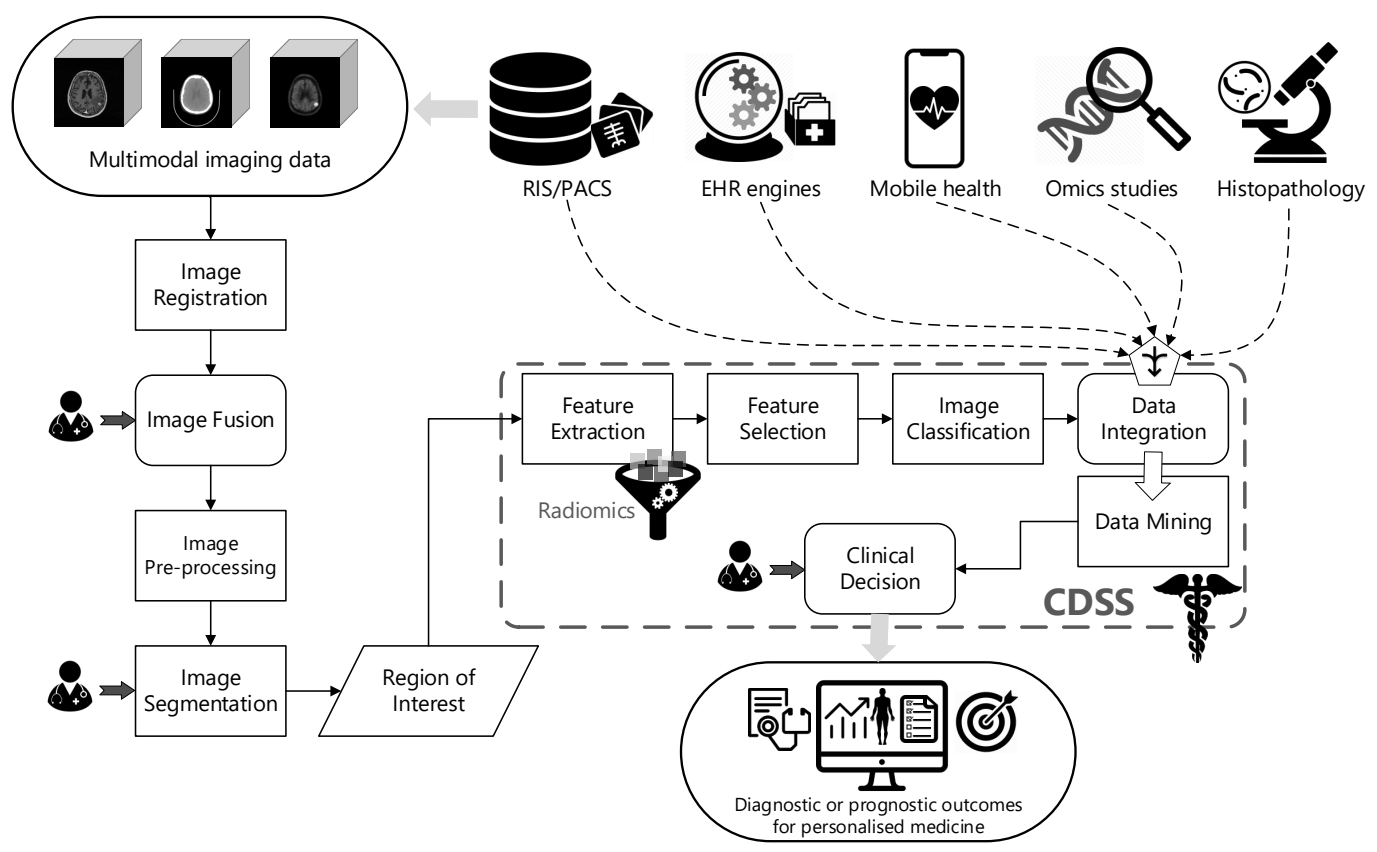

Figure 1. Workflow that integrates nature-inspired computational methods into the clinical routine. Especially, an intelligent CDSS is used to properly combine and analyse several sources of information to support the physicians in their decision-making tasks. The steps that may require the physician's interaction are also highlighted.

In current clinical routine, quantitative imaging methods convey scientifically and clinically relevant information in prediction, prognosis or treatment response assessment [14], by also considering radiomics approaches [15]. In this regard, advanced computational techniques play a key role in radiology [16]. However, conventional Machine Learning and Computational Intelligence techniques must be tailored to address the specific challenges pertaining to biomedical images [17]. Relevant models, features, and characteristics can be indeed automatically learnt from images by taking advantage of these computational frameworks realised for medical imaging problems involving advanced data integration.

\subsection{Integrating nature-inspired methods into the clinical workflow}

The principal contribution of research in computer science to biomedical data integration concerns the proper fusion of diverse and heterogeneous datasets [18, 19]-i.e., medical imaging modalities (possibly validating radiomics-based biomarkers against histopathology [20]), Electronic Health Record (EHR) engines [21], high-throughput technologies (i.e., multi-omics studies [22]), and real-time monitoring (exploiting m-health applications) — to provide a comprehensive clinical knowledge for precision medicine [9]. Fig. 1 outlines the steps involved in the clinical decision making-process. Natureinspired computational techniques can be suitably applied to every phase of this pipeline tightly cou- 


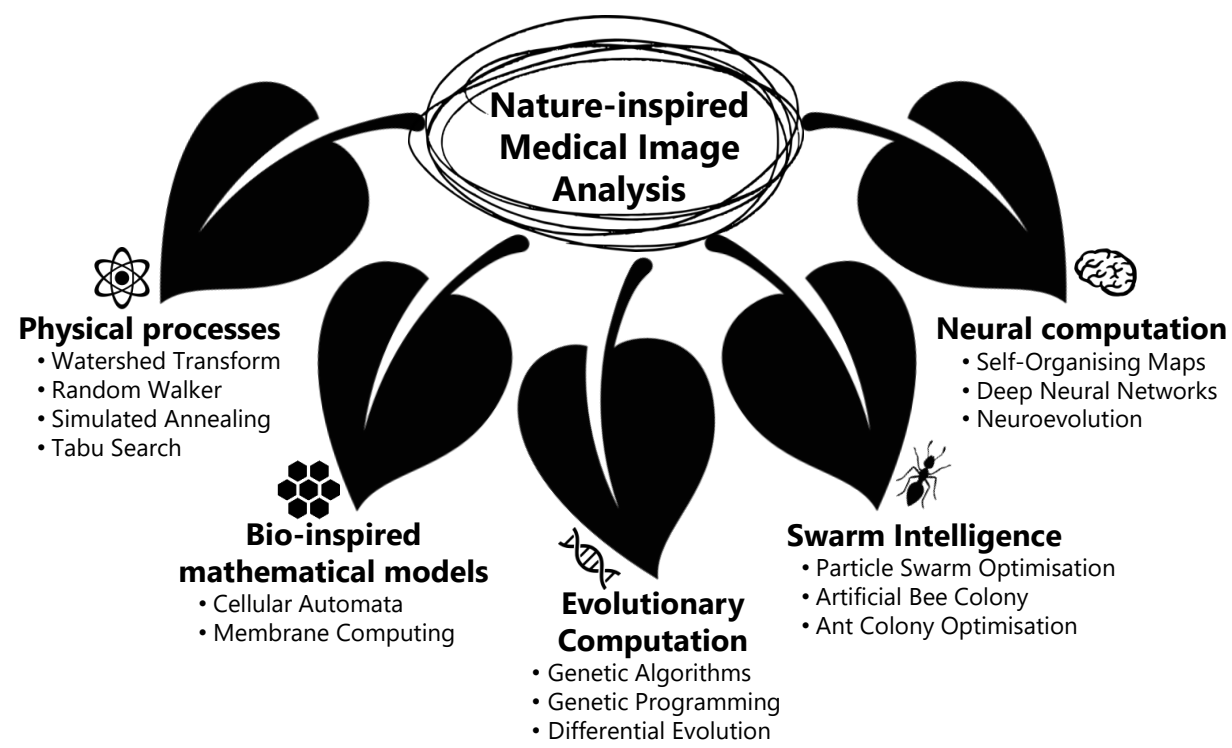

Figure 2. Taxonomy of the investigated nature-inspired computational techniques for medical image analysis. Each branch, denoted by a different leaf, corresponds to a class of algorithms that is described in a different section of this manuscript.

pled with Clinical Decision Support Systems (CDSSs) as well as physician's interaction. As a matter of fact, this biomedical data-oriented paradigm needs for novel inference tools, able to deal with complex/rare diseases and comorbidities [23].

In this context, medical image analysis involves interdisciplinary research activities carried out by computer scientists, physicians, and medical physicists. The multimodal imaging data as well as the corresponding patient information are retrieved from the Picture Archiving and Communication System (PACS) interconnected with the Radiology Information System (RIS). Especially, image fusion aligns and combines multiple images from single or multiple imaging modalities, aiming at improving the image quality as well as reducing randomness and redundancy, towards feasible solutions for improved diagnosis and prognosis [24].

\subsection{Taxonomy of the investigated nature-inspired computational techniques}

This review outlines the state-of-the-art of nature-inspired medical image analysis methods, aiming at establishing a common platform for beneficial exchanges among computer scientists and clinicians. To achieve a proper level of generality, we focus on the main nature-inspired computational techniques applied to medical image analysis tasks. The organisation of the investigated approaches follows the graphical representation in Fig. 2. This conceptual scheme shows that the appropriate combination of different computational techniques can shed light on new discoveries in healthcare and biology. Therefore, the synergies emerging from the combination of diverse computer science areas are valuable to develop comprehensive approaches in clinical routine. These computer-assisted medical image anal- 


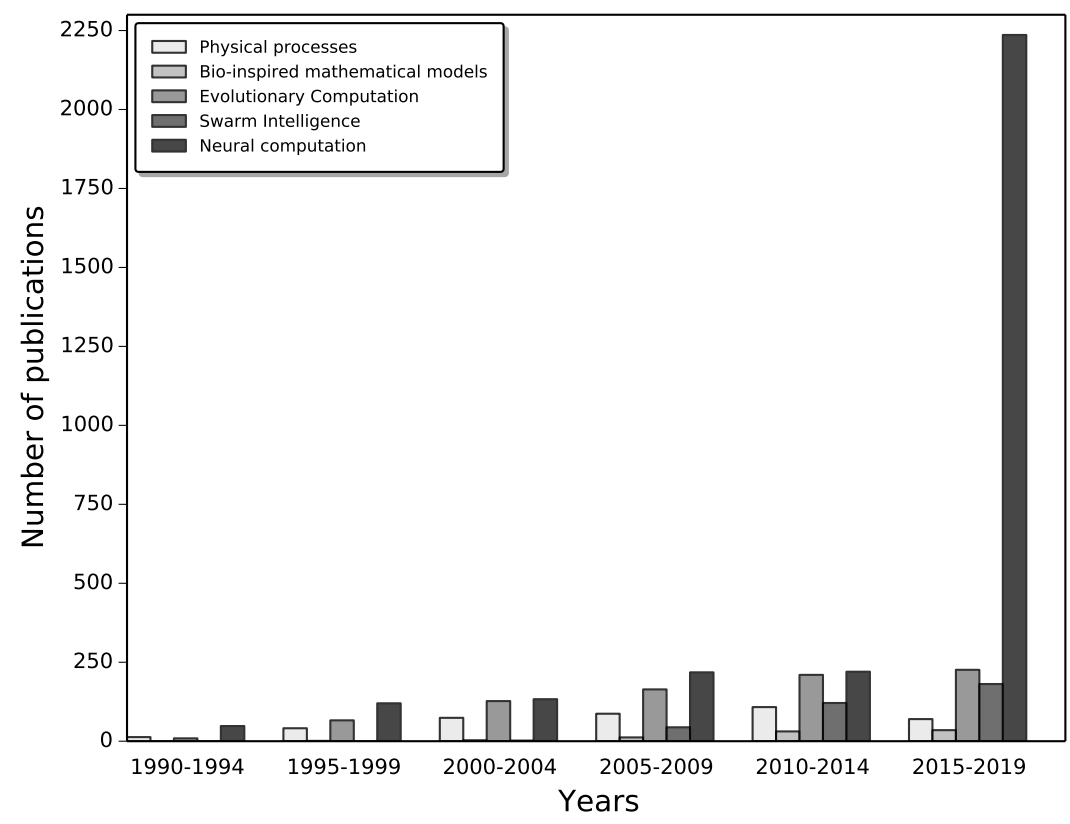

Figure 3. Bar diagram of the number of publications sub-divided by each different class of the nature-inspired techniques schematised in Fig. 2 and grouped by 5 -year intervals (ranging from 1990 to 2019). The number of papers for 2019 refers to the date of writing (March 2019).

ysis methods could be beneficial for the definition of imaging biomarkers as well as for quantitative medicine and biology.

The structure of this manuscript reflects the different branches depicted in Fig. 2, by providing some interesting medical imaging applications. Section 2 describes the methods based on physical processes occurring in nature. Section 3 presents bio-inspired mathematical models, with particular interest to unconventional and Natural Computing. Computational Intelligence techniques, consisting in Evolutionary Computation and Swarm Intelligence, are devised in Sections 4 and 5, respectively. The most recent neural computation models are explained in Section 6. Finally, discussions and concluding remarks are provided in Section 7.

Aiming at providing the reader with a clear idea about the development of the nature-inspired techniques in the field of medical image analysis, a bar diagram representing the use of the different techniques throughout the years is shown in Fig. 3. The number of contributions (considering 5 -year intervals from 1990 to 2019) was obtained by relying on Scopus ${ }^{\circledR}$ _a well-known database of peerreviewed literature - for articles matching separate queries composed of the logical disjunction (i.e., OR of all the components for each branch in Fig. 2) AND "medical imaging" OR "medical image analysis" OR "biomedical images". The general trend reveals a considerable growth in the adoption of nature-inspired computational techniques over the years. In particular, Evolutionary Computation has been remarkably employed in medical image analysis since the early 2000s also reaching the popularity of neural approaches in 2010-2014, despite the traditional applications of Artificial Neural 
Networks (ANNs) in Pattern Recognition. However, in the last 5 years, the most noticeable aspect is the striking increase of works based on neural computation, almost entirely due to the success of Deep Learning. As a matter of fact, this breakthrough is tightly linked to the evidence that the number of articles presenting neural-based methods doubles approximately every year since 2015 .

\section{Physical processes}

The concepts underlying physical phenomena can lead to valuable ideas in algorithm design. We explain some basic theoretical models in the following sub-sections.

\subsection{Watershed transform}

The watershed transform, initially proposed in the field of mathematical morphology [25], intuitively denotes an image as a topographic relief, where the height of each point is directly related to its grey level. Considering rain gradually falling on the terrain, the watersheds are the lines that separate the resulting catchment basins by relying on a flooding-based procedure [26]. The watershed lines generally correspond to the most significant edges among the markers [27] and are useful to separate overlapping objects, such as adjacent cells in tissues. Even when no strong edges between the markers exist, the watershed method is able to detect a contour in the area. This contour is detected on the pixels with higher contrast [25]. Unfortunately, it is also well-known that the watershed transform may be affected by over-segmentation issues, so requiring further processing. The authors of [28] introduced probability-based prior information into the watershed segmentation for knee cartilage and grey matter/white matter segmentation in MR images.

\subsection{Random Walker}

The Random Walker (RW) algorithm [29] encodes an image as a graph with nodes and arcs corresponding to the voxels and changes in image intensity (denoted by means of a Gaussian function), respectively. Therefore, the image is converted into a lattice where some pixels' classes are known while other ones are unassigned. Random walks enable a model for Brownian motion and diffusion, e.g., the stochastic movement of molecules in liquids and gases. In medical imaging, especially when dealing with Positron Emission Tomography (PET), the RW method can localise weak edges as part of consistent boundaries. To obtain the Biological Target Volume (BTV) delineation on metabolic PET images, the RW incorporates the PET information, by replacing the pixel intensities with the Standardised Uptake Value (SUV) and computing the arc weights accordingly [30]. In the original method [29], the known nodes are marked by user input, whilst an automatic seed-selection procedure makes the method more reliable [31]. The study presented in [32] investigated the impact of BTV segmentation, using $\left[{ }^{11} \mathrm{C}\right]-$ Methionine PET imaging, and the subsequent co-registration with Magnetic Resonance Imaging (MRI), utilised to delineate the Gross Tumour Volume (GTV) [33, 34], in stereotactic neuro-radiosurgery treatment planning. The main goal was to present a novel PET/MRI automatic segmentation method, combining complementary multimodal information, and encouraging its use in clinical practice. 


\subsection{Simulated Annealing and Tabu Search}

Neighbourhood-based meta-heuristics, such as Simulated Annealing (SA) [35] and Tabu Search (TS) [36], evaluate only one candidate solution at a time across the search space [4]. The SA method was initially designed for combinatorial optimisation problems and emulates the annealing process of solid materials, whose temperature is initially increased to high values and then slowly cooled by a controlled procedure to dilate the crystals and reduce their defects. An efficient version of SA coupled with an adaptive Monte Carlo sampling algorithm was proposed in [37] for the deformable registration of 4D Computed Tomography (CT) lung images. TS is based on adaptive memory-based strategies to guide the search towards promising areas as well as discourage the search from previouslyvisited solutions to improve the diversity. Wachowiack et al. [38] applied the continuous TS to the co-registration of $2 \mathrm{D}$ Ultrasound scans to $3 \mathrm{D}$ volumes.

\section{Bio-inspired mathematical models}

Natural Computing devises mathematical and unconventional computation models inspired by nature, as well as investigates phenomena taking place in nature in terms of information processing [39].

\subsection{Cellular Automata}

Cellular Automata (CA) are one of the earliest models of bio-inspired computing [40]. Discrete CA are composed of self-replicating artificial systems in a mesh of finite-state machines (cells), interconnected locally to each other, which produce a complex global emergent behaviour [13]. Each cell changes its own state synchronously depending on both: (i) its current state, and (ii) the states of the neighbour cells at the previous discrete time step. All cells use the same local update rule, so defining a homogeneous system [41]. Hamamci et al. presented Tumor-Cut [42] that employs a CA model implementing the gradient magnitude as the local transition rule, resulting equivalent to the shortest path algorithm, as shown in [43]. Analogously, GTVCUT is a CA-based brain tumour MRI segmentation method designed for neuro-radiosurgery treatment planning [44, 45]. This semi-automatic segmentation approach adaptively initialises the foreground and background seeds in a smart fashion, relying only on a draggable rectangle including the target area [46].

\subsection{Membrane Computing}

Membrane Computing is an unconventional model of computation that assumes the functioning of the cells as an information processing system. In particular, this model formalises the flow of metabolites among cells of a living tissue or among the organelles in an eucaryotic cell. More specifically, the computational devices in Membrane Computing are called P systems [47]. This model can be efficiently implemented on parallel architectures [48], and have been applied to image segmentation [49]. P systems were efficiently applied also to edge detection and multi-level thresholding in [50] and [51], respectively. 


\section{Evolutionary Computation}

Evolutionary Computation, taking inspiration from Darwin's theory of biological evolution [52], includes population-based optimisation techniques that solve complex real-world problems that cannot be tackled by means of exact algorithmic approaches. In clinical scenarios, multi-objective evolutionary algorithms were applied to high-dose-rate brachytherapy planning for prostate cancer treatment [53], wherein clinical protocols involving multiple different treatment criteria as well as time constraints must be satisfied [54].

\subsection{Genetic Algorithms}

Genetic Algorithms (GAs) rely on natural selection: the initial population of randomly generated individuals undergoes, during each generation, a selection process and is modified by applying crossover and mutation operators [55]. A novel evolutionary method based on GAs for medical image enhancement and segmentation, named MedGA, was recently presented in [56], by using the efficient histogram-based encoding of individuals defined in [57]. MedGA aims at better revealing the two underlying sub-distributions occurring in a medical image sub-region characterised by a roughly bimodal histogram, in order to improve thresholding-based segmentation results [58]. An extensive review of the applications of GAs to medical image segmentation is presented in [59]. Indeed, GAs can be conveniently applied to diverse segmentation frameworks. Cagnoni et al. [60] proposed an interactive contour-based delineation model for anatomical structures in tomographic medical images, where a GA drives the elastic-contour evolution. With reference to multi-atlas based segmentation, GAs were employed to select the near-optimal atlas sub-set combination to segment the current target image [61]. GAs are also effective for the selection of relevant and informative radiomics features [62], aiming at improving the discriminative power for subsequent classification tasks. In [63], GAs are exploited for the feature selection phase to improve breast cancer diagnosis on digitised fine-needle aspiration cytology images by comparing multiple classifiers.

\subsection{Genetic Programming}

Genetic Programming (GP) [64] was shown to be a powerful framework to select and combine existing algorithms in the most suitable way, also in Computer Vision [65]. Unlike GAs, GP evolves a population of functions or, more generally, computer programs to solve a problem. In the medical domain, GP with geometrics semantic operators [66] was employed to predict the relative position of a slice within a CT image stack when DICOM metadata are unreliable [67]. With regard to GPbased image enhancement, optimal pseudo-colouring of multiple gray-scale images for visualisation purposes was addressed in [68], by relying on user interaction in the tournament selection.

\subsection{Differential Evolution}

Differential Evolution (DE) exploits crossover and mutation operators during the evolutionary process [69]. Unlike GAs, DE randomly mutates an individual and then employs a ternary crossover operator 
by recombining three individuals selected randomly from the population. Moreover, in DE the competition mechanism involves only the offspring along with their parents, not the whole population. In [70], a DE-based reversible watermarking scheme for improving the security of storage and exchange of medical images was proposed. Here, signature and textual information is inserted into the original images, exploiting DE in the optimal quantisation step for the control of watermarking strength.

\section{Swarm Intelligence}

Swarm Intelligence studies the collective behaviour of decentralised, self-organised natural or artificial systems [71]. These metaheuristics consist typically of a population of simple agents interacting locally each other and with their environment. The agents follow very simple rules and, although there is no centralised control structure, local interactions among such agents - often affected by a certain degree of stochasticity-lead to a complex intelligent emergent global behaviour, with effects that would not have been expected by each individual.

\subsection{Particle Swarm Optimisation}

Particle Swarm Optimisation (PSO), which is inspired by the collective movement of bird flocks and fish schools, is well-suited for solving non-linear optimisation problems [72]. Formally, a swarm of individuals (i.e., particles) moves inside a bounded $D$-dimensional search space cooperating to identify the optimal solution, by collecting and sharing information about the best position found according to a user-defined fitness function. The movement of particles in the search space is modelled by the social and cognitive components in the velocity update rules. Since PSO was originally introduced for continuous search spaces, it can be applied to the optimisation of similarity metrics (e.g., cross correlation, mutual information) in biomedical image registration [73]. Wachowiak et al. [74] devised a modified version of PSO including crossover operations as well as the initial orientation of the volumes exploiting the knowledge by the physician to considerably improve the search process. Finally, in [75], a hybrid approach is proposed, which integrates the spatial image information into the computation of the similarity metrics by means of a linear combination of image pixel intensity and image Gradient Vector Flow intensity.

\subsection{Artificial Bee Colony}

The Artificial Bee Colony (ABC) algorithm is based on the foraging behaviour of honey bees [76], which cooperate in identifying the best food resources corresponding to the solutions with the best fitness values. Specifically, the swarm is composed of three specialised groups of honey bees, namely: scouts, onlookers, and employees. The scouts are randomly distributed across the search space, while employees and onlookers accomplish a local search nearby the promising positions found by the scouts. In [77], ABC was effectively exploited for global multi-level thresholding method based on the Tsallis entropy as segmentation criterion. 


\subsection{Ant Colony Optimisation}

Ant Colony Optimisation (ACO) is a metaheuristic that resembles the foraging behaviour of some ant species [78]. The artificial ants are stochastic procedures that incrementally construct candidate solutions for the problem instance under consideration by exploiting the pheromone information that is adapted according to the ants' search experience and possible available heuristic information [79]. Pereira et al. [80] proposed an ACO-based method for optic disc detection in colour fundus images. An adaptive ACO-based segmentation algorithm was designed in [81] for breast cancer diagnosis on MRI, explicitly considering also object brightness and circularity. These features are useful for an accurate selection of the detected lesions.

\section{Neural computation}

Neural computation is based on theoretical and computational neurosciences, aiming at modelling the information processing that occurs in networks of biological neurons [82]. Several types of ANNs are explained in what follows.

\subsection{Self-Organising Maps}

Self-Organising Maps (SOMs) rely on neuro-biological studies indicating that different sensory inputs (e.g., motor, visual, auditory) are mapped onto corresponding areas of the cerebral cortex in a certain order [83]. Essentially, SOMs aim at building artificial topographic maps that learn by means of selforganisation, allowing for short synaptic connections for neurons dealing with closely-related portions of information. This technique is well-suited for dimensionality reduction, so enabling effective data visualisation and cluster analysis. Ortiz et al. [62] applied a SOM-based classification method for brain tissue segmentation on MR images.

\subsection{Deep Neural Networks}

In the latest years, Deep Neural Networks (DNNs) have been widely exploited to learn a hierarchy of increasingly complex features from the processed data, enabling multiple levels of abstraction [84]. Specifically, DNNs exploit the property that many natural signals are compositional hierarchies (i.e., higher-level features are obtained by composing lower-level ones). Hereby, we describe two classes of DNNs that are successfully applied to medical imaging.

\subsubsection{Convolutional Neural Networks}

Although in traditional multi-layer networks each neuron is densely or fully connected to every neuron of the subsequent layer, considering only local sub-structural information could be useful in Pattern Recognition. Relying on the initial idea of the neocognitron [85], neighbouring pixels in the image were shown to be strongly correlated. Thus, they can represent meaningful features such as edges. On the contrary, distant pixels tend to be weakly correlated or even uncorrelated. These architectures, called Convolutional Neural Networks (CNNs), are deep feed-forward ANNs that are suitable 
to work on data structured with a grid-like topology, such as images, videos, and time-series. The convolutional and pooling layers in CNNs are directly inspired by the classic notions of simple cells and complex cells in visual neuroscience [86]. Considering the most recent computational methods in medical image segmentation, significant advances have been proposed in CNN-based architectures [87]. For instance, to overcome the limitations related to accurate image annotations, DeepCut [88] relies on weak bounding-box labelling $[46,45]$. Among the architectures devised for biomedical image segmentation $[89,90]$, U-Net [91] showed to be a noticeably successful solution, thanks to the combination of a contracting path, for coarse-grained context detection, and a symmetric expanding path, for fine-grained localisation. This fully CNN is capable of stable training with reduced samples. The authors of V-Net [92] extended U-Net for volumetric medical image segmentation, by introducing also a different loss function based on the Dice Similarity Coefficient. Schlemper et al. [93] presented an Attention Gate (AG) model for medical imaging, which aims at focusing on target structures or organs. AGs were introduced into the standard U-Net, so defining Attention U-Net, which achieved high performance in multi-class image segmentation without relying on multi-stage cascaded CNNs. Recently, Mixed-Scale Dense (MS-D) Net [94] densely connected the features at different scales obtained by means of dilated convolutions. By so doing, features at different scales can be contextually extracted using fewer parameters than fully CNNs.

\subsubsection{Generative Adversarial Networks}

Generative Adversarial Networks (GANs) introduce a framework for unsupervised to estimate generative models via an adversarial process by simultaneously training two models [95]. Originally proposed by Goodfellow et al. in 2014 [95], GANs have shown remarkable results in image generation [96] relying on a two-player minimax game: a generator network aims at generating realistic images to "fool" a discriminator network that aims at distinguishing between the real and synthetic images. However, the resulting objective function could lead to difficult training, accompanying artifacts and mode collapse [97], especially with high resolution images. Even though CNNs have recently revolutionised medical image analysis [12], their training requires large-scale annotated training data that are laborious to obtain in the medical domain [98]; thus, intensive Data Augmentation (DA) techniques, such as geometric/intensity transformations of original images, are needed. However, those reconstructed images intrinsically resemble the original ones, leading to limited performance improvement in terms of generalisation abilities. Therefore, the generation of realistic medical images completely different from the original samples is worth to investigate. Realistic retinal fundus and lung CT image generation tasks were tackled using adversarial learning [99, 100]; a recent study reported performance improvement with synthetic training data in CNN-based liver lesion classification [101]. In [102], brain tumour MR images were generated using conventional GANs and even an expert physician failed to accurately distinguish between the real/synthetic images. The goal was to increase the clinical reliability, by means of DA in computer-assisted diagnosis as well as physician training and teaching [103]. However, this objective is extremely challenging, since MR images are characterised by strong visual consistency in brain anatomy, and intra-sequence variability. 


\subsection{Neuroevolution}

Neuroevolution - i.e., the evolutionary-based automatic design of ANNs [104]—resembles the evolution of biological brain, which may contain trillions of synaptic connections. Specifically, HyperNEAT [105] is a generative encoding that evolves large-scale ANNs relying on the NeuroEvolution of Augmented Topologies (NEAT) algorithm [106], which search for the geometric regularities of the task domain. Calimeri et al. [107] applied HyperNEAT to the automated segmentation of blood vessels in digital retinal fundus images. This method obtained promising results, revealing the ability to reduce the size of ANNs for medical image segmentation tasks.

\section{Conclusions and future directions}

Quantitative studies in biomedicine represent the immediate future in clinical practice [14, 108]. The proper combination of different imaging modalities and healthcare informatics provides an in-depth understanding of molecular processes that can guide towards personalised medicine [109].

The achievements related to quantitative imaging lead to valuable applications in radiomics [15, $110,111,112]$ and radiogenomics $[113,114]$ research, thus conveying clinically useful information. Especially, radiogenomics [114] can provide important information about tumour heterogeneity as well as treatment response [115], by also integrating genomic information [116]. In this regard, personalised medicine may definitely benefit from advanced nature-inspired computational approaches, such as in the case of haplotype assembly based on GAs [117].

The integration of Soft Computing, including rough sets [118] and fuzzy logic [119], may properly deal with the vagueness and coarseness in medical image analysis tasks (e.g., image segmentation $[120,121])$. In the case of global optimisation methods for biomedical image registration, a set of fuzzy rules may be exploited to dynamically adapt the settings for each particle of the PSO, so resulting in proactive optimising agents [122], achieving encouraging performance on benchmark functions [123] as well as in the parameter estimation of biochemical systems [124]. Concerning other unconventional computation models, Quantum Computing — which studies the information processing tasks executed on quantum mechanical systems [125] — might be applied to basic and advanced medical image processing operations, by devising effective techniques of internal representation of the images involved in a quantum process [126].

From the technological perspective, High-Performance Computing (HPC) can be an enabling factor for feasible computational solutions in clinical and laboratory practice [127]. GPUs are used today in a wide range of applications in computational biology [128] and medical image analysis [129, 130]. As a matter of fact, GPUs are pervasive, energy-efficient and can dramatically accelerate parallel computing. In the field of biomedical imaging, GPUs are in some cases crucial for enabling practical use of computationally demanding algorithms. For instance, GPUs enabled the training of DNNs in reasonable time $[12,131]$. Finally, the community of computer scientists and engineers in biomedicine cannot ignore the interpretability of the results yielded by advanced computational models [132]. As a matter of fact, the outcomes and predictions provided by CDSSs should take into account the physicians' awareness during their complex clinical decision-making tasks [133]. Considering the unique challenges encountered in clinical scenarios, also the issues related to the interaction design of medical 
imaging software must be properly addressed. Indeed, computer applications for diagnostic medical imaging generally provide a wide range of tools to support physicians in their daily diagnosis activities. Unfortunately, some functionalities are specialised for specific diseases or imaging modalities, while others are useless for the images under investigation [134]. In clinical environments, a Graphical User Interface (GUI) must represent a sequence of steps for image investigation following a well-defined workflow.

\section{Acknowledgements}

This work was partially supported by The Mark Foundation for Cancer Research and Cancer Research UK Cambridge Centre [C9685/A25177].

\section{References}

[1] Mitra S, Shankar BU. Medical image analysis for cancer management in natural computing framework. Inform. Sci., 2015. 306:111-131. doi:10.1016/j.ins.2015.02.015.

[2] Kar AK. Bio inspired computing-a review of algorithms and scope of applications. Expert Syst. Appl., 2016. 59:20-32. doi:10.1016/j.eswa.2016.04.018.

[3] Hidalgo-Herrero M, Rabanal P, Rodriguez I, Rubio F. Comparing problem solving strategies for NP-hard optimization problems. Fundam. Inform., 2013. 124(1-2):1-25. doi:10.3233/FI-2013-822.

[4] Siddique N, Adeli H. Nature inspired computing: an overview and some future directions. Cogn. Computat., 2015. 7(6):706-714. doi:10.1007/s12559-015-9370-8.

[5] Peng H. Bioimage informatics: a new area of engineering biology. Bioinformatics, 2008. 24(17):18271836. doi:10.1093/bioinformatics/btn346.

[6] Shamir L, Delaney JD, Orlov N, Eckley DM, Goldberg IG. Pattern recognition software and techniques for biological image analysis. PLoS Comput. Biol., 2010. 6(11):e1000974. doi:10.1371/journal.pcbi. 1000974.

[7] Gray JE, Feldman H, Reti S, Markson L, Lu X, Davis RB, Safran CA. Using Digital Crumbs from an Electronic Health Record to identify, study and improve health care teams. In: AMIA Annu. Symp. Proc., volume 2011. American Medical Informatics Association, 2011 p. 491.

[8] Alepis E, Lambrinidis C. M-health: supporting automated diagnosis and electronic health records. SpringerPlus, 2013. 2(1):103. doi:10.1186/2193-1801-2-103.

[9] Brady M, Highnam R, Irving B, Schnabel JA. Oncological image analysis. Med. Image Anal., 2016. 33:7-12. doi:10.1016/j.media.2016.06.012.

[10] Serbanati LD, Ricci FL, Mercurio G, Vasilateanu A. Steps towards a digital health ecosystem. J. Biomed. Inform., 2011. 44(4):621-636. doi:10.1016/j.jbi.2011.02.011.

[11] Meijering E, Carpenter AE, Peng H, Hamprecht FA, Olivo-Marin JC. Imagining the future of bioimage analysis. Nat. Biotechnol., 2016. 34(12):1250. doi:10.1038/nbt.3722.

[12] Shen D, Wu G, Suk HI. Deep learning in medical image analysis. Annu. Rev. Biomed. Eng., 2017. 19:221-248. doi:10.1146/annurev-bioeng-071516-044442. 
[13] Kari L, Rozenberg G. The many facets of natural computing. Commun. ACM, 2008. 51(10):72-83. doi:10.1145/1400181.1400200.

[14] Yankeelov TE, Mankoff DA, Schwartz LH, Lieberman FS, Buatti JM, Mountz JM, Erickson BJ, Fennessy FM, Huang W, Kalpathy-Cramer J, et al. Quantitative imaging in cancer clinical trials. Clin. Cancer Res., 2016. 22(2):284-290. doi:10.1158/1078-0432.CCR-14-3336.

[15] Aerts HJ, Velazquez ER, Leijenaar RT, Parmar C, Grossmann P, Carvalho S, Bussink J, Monshouwer R, Haibe-Kains B, Rietveld D, et al. Decoding tumour phenotype by noninvasive imaging using a quantitative radiomics approach. Nat. Commun., 2014. 5:4006.

[16] Wernick MN, Yang Y, Brankov JG, Yourganov G, Strother SC. Machine learning in medical imaging. IEEE Signal Process. Mag., 2010. 27(4):25-38. doi:10.1109/MSP.2010.936730.

[17] Hosny A, Parmar C, Quackenbush J, Schwartz LH, Aerts HJ. Artificial intelligence in radiology. Nat. Rev. Cancer, 2018. 18(8):500-510. doi:10.1038/s41568-018-0016-5.

[18] Lahat D, Adali T, Jutten C. Multimodal data fusion: an overview of methods, challenges, and prospects. Proc. IEEE, 2015. 103(9):1449-1477. doi:10.1109/JPROC.2015.2460697.

[19] Serra A, Galdi P, Tagliaferri R. Machine learning for bioinformatics and neuroimaging. Wiley Interdisc. Rev. Data Min. Knowl. Discov., 2018. p. e1248. doi:10.1002/widm.1248.

[20] Fehr D, Veeraraghavan H, Wibmer A, Gondo T, Matsumoto K, Vargas HA, Sala E, Hricak H, Deasy JO. Automatic classification of prostate cancer Gleason scores from multiparametric magnetic resonance images. Proc. Natl. Acad. Sci., 2015. 112(46):E6265-E6273. doi:10.1073/pnas.1505935112.

[21] Alaa AM, Moon KH, Hsu W, van der Schaar M. ConfidentCare: a clinical decision support system for personalized breast cancer screening. IEEE Trans. Multimed., 2016. 18(10):1942-1955. doi:10.1109/ TMM.2016.2589160.

[22] Libbrecht MW, Noble WS. Machine learning applications in genetics and genomics. Nat. Rev. Genet., 2015. 16(6):321. doi:10.1038/nrg3920.

[23] Capobianco E, Liò P. Comorbidity networks: beyond disease correlations. J. Complex Netw., 2015. 3(3):319-332. doi:10.1093/comnet/cnu048.

[24] James AP, Dasarathy BV. Medical image fusion: a survey of the state of the art. Inform. Fusion, 2014. 19:4-19. doi:10.1016/j.inffus.2013.12.002.

[25] Soille PJ, Ansoult MM. Automated basin delineation from digital elevation models using mathematical morphology. Signal Process., 1990. 20(2):171-182. doi:10.1016/0165-1684(90)90127-K.

[26] Vincent L, Soille P. Watersheds in digital spaces: an efficient algorithm based on immersion simulations. IEEE Trans. Pattern Anal. Mach. Intell., 1991. 13(6):583-598. doi:10.1109/34.87344.

[27] Beucher S, Meyer F. The morphological approach to segmentation: the watershed transformation. In: Mathematical Morphology in Image Processing, volume 34, pp. 433-481. Marcel Dekker Inc., New York. NY, USA, 1993.

[28] Grau V, Mewes A, Alcaniz M, Kikinis R, Warfield SK. Improved watershed transform for medical image segmentation using prior information. IEEE Trans. Med. Imaging, 2004. 23(4):447-458. doi: 10.1109/TMI.2004.824224.

[29] Grady L. Random walks for image segmentation. IEEE Trans. Pattern Anal. Mach. Intell., 2006. 28(11):1768-1783. doi:10.1109/TPAMI.2006.233. 
[30] Stefano A, Vitabile S, Russo G, Ippolito M, Marletta F, D’Arrigo C, D’Urso D, Gambino O, Pirrone $\mathrm{R}$, Ardizzone E, et al. A fully automatic method for biological target volume segmentation of brain metastases. Int. J. Imaging Syst. Technol., 2016. 26(1):29-37. doi:10.1002/ima.22154.

[31] Stefano A, Vitabile S, Russo G, Ippolito M, Sabini MG, Sardina D, Gambino O, Pirrone R, Ardizzone E, Gilardi MC. An enhanced random walk algorithm for delineation of head and neck cancers in PET studies. Med. Biol. Eng. Comput., 2017. 55(6):897-908. doi:10.1007/s11517-016-1571-0.

[32] Rundo L, Stefano A, Militello C, Russo G, Sabini MG, D’Arrigo C, Marletta F, Ippolito M, Mauri G, Vitabile S, Gilardi MC. A fully automatic approach for multimodal PET and MR image segmentation in Gamma Knife treatment planning. Comput. Methods Programs Biomed., 2017. 144:77-96. doi: 10.1016/j.cmpb.2017.03.011.

[33] Militello C, Rundo L, Vitabile S, Russo G, Pisciotta P, Marletta F, Ippolito M, D’Arrigo C, Midiri M, Gilardi MC. Gamma Knife treatment planning: MR brain tumor segmentation and volume measurement based on unsupervised fuzzy c-means clustering. Int. J. Imaging Syst. Technol., 2015. 25(3):213-225. doi:10.1002/ima.22139.

[34] Rundo L, Militello C, Vitabile S, Russo G, Pisciotta P, Marletta F, Ippolito M, DArrigo C, Midiri M, Gilardi MC. Semi-automatic brain lesion segmentation in Gamma Knife treatments using an unsupervised fuzzy c-means clustering technique. In: Advances in Neural Networks: Computational Intelligence for ICT, volume 54 of Smart Innovation, Systems and Technologies, pp. 15-26. Springer, 2016. doi:10.1007/978-3-319-33747-0_2.

[35] Kirkpatrick S, Gelatt CD, Vecchi MP. Optimization by simulated annealing. Science, 1983. 220(4598):671-680. doi:10.1126/science.220.4598.671.

[36] Glover F. Future paths for integer programming and links to artificial intelligence. Comput. Oper. Res., 1986. 13(5):533-549. doi:10.1016/0305-0548(86)90048-1.

[37] Dou TH, Min Y, Neylon J, Thomas D, Kupelian P, Santhanam AP. Fast simulated annealing and adaptive Monte Carlo sampling based parameter optimization for dense optical-flow deformable image registration of 4DCT lung anatomy. In: Medical Imaging 2016: Image-Guided Procedures, Robotic Interventions, and Modeling, volume 9786 of Prof. SPIE. International Society for Optics and Photonics, 2016 p. 97860N. doi:10.1117/12.2217194.

[38] Wachowiak MP, Elmaghraby AS. The continuous tabu search as an optimizer for 2D-to-3D biomedical image registration. In: Proc. International Conference on Medical Image Computing and ComputerAssisted Intervention (MICCAI), volume 2208 of LNCS. Springer, 2001 pp. 1273-1274. doi:10.1007/ 3-540-45468-3_191.

[39] Rozenberg G, Bck T, Kok JN. Handbook of Natural Computing. Springer Publishing Company, Incorporated, Berlin-Heidelberg, Germany, 1 edition, 2011. ISBN 978-3-540-92909-3.

[40] Von Neumann J, Burks AW. Theory of self-reproducing automata. University of Illinois Press, Urbana, IL, USA, 1966.

[41] Kari J. Theory of cellular automata: a survey. Theor. Comput. Sci., 2005. 334(1-3):3-33. doi:10.1016/j. tcs.2004.11.021.

[42] Hamamci A, Kucuk N, Karaman K, Engin K, Unal G. Tumor-Cut: segmentation of brain tumors on contrast enhanced MR images for radiosurgery applications. IEEE Trans. Med. Imaging, 2012. 31(3):790804. doi:10.1109/TMI.2011.2181857. 
[43] Sinop AK, Grady L. A seeded image segmentation framework unifying graph cuts and random walker which yields a new algorithm. In: 11th International Conference on Computer Vision (ICCV) 2007. IEEE, 2007 pp. 1-8. doi:10.1109/ICCV.2007.4408927.

[44] Rundo L, Militello C, Russo G, Pisciotta P, Valastro LM, Sabini MG, Vitabile S, Gilardi MC, Mauri G. Neuro-radiosurgery treatments: MRI brain tumor seeded image segmentation based on a cellular automata model. In: Proc. 12th International Conference on Cellular Automata for Research and Industry (ACRI), volume 9863 of LNCS. Springer, 2016 pp. 323-333. doi:10.1007/978-3-319-44365-2_32.

[45] Rundo L, Militello C, Russo G, Vitabile S, Gilardi MC, Mauri G. GTVcut for neuro-radiosurgery treatment planning: an MRI brain cancer seeded image segmentation method based on a cellular automata model. Nat. Comput., 2018. 17(3):521-536. doi:10.1007/s11047-017-9636-z.

[46] Rother C, Kolmogorov V, Blake A. GrabCut: interactive foreground extraction using iterated graph cuts. ACM Trans. Graph., 2004. 23(3):309-314. doi:10.1145/1186562.1015720.

[47] Păun G. Computing with membranes. J. Comput. Syst. Sci., 2000. 61(1):108-143. doi:10.1006/jcss. 1999.1693.

[48] Nobile MS, Porreca AE, Spolaor S, Manzoni L, Cazzaniga P, Mauri G, Besozzi D. Efficient simulation of reaction systems on Graphics Processing Units. Fundam. Inform., 2017. 154(1-4):307-321. doi: 10.3233/FI-2017-1568.

[49] Yahya RI, Shamsuddin SM, Yahya SI, Hasan S, Al-Salibi B, Al-Khafaji G. Image segmentation using membrane computing: a literature survey. In: Gong M, Pan L, Song T, Zhang G (eds.), Bio-Inspired Computing - Theories and Applications (BIC-TA), volume 681 of Communications in Computer and Information Science. Springer, 2016 pp. 314-335. doi:10.1007/978-981-10-3611-8_26.

[50] Díaz-Pernil D, Berciano A, Peña-Cantillana F, Gutiérrez-Naranjo MA. Segmenting images with gradientbased edge detection using membrane computing. Pattern Recogn. Lett., 2013. 34(8):846-855. doi: 10.1016/j.patrec.2012.10.014.

[51] Peng H, Wang J, Pérez-Jiménez MJ, Shi P. A novel image thresholding method based on membrane computing and fuzzy entropy. J. Intell. Fuzzy Syst., 2013. 24(2):229-237. doi:10.3233/IFS-2012-0549.

[52] Eiben AE, Smith J. From evolutionary computation to the evolution of things. Nature, 2015. 521(7553):476-482. doi:10.1038/nature14544.

[53] Rundo L, Militello C, Russo G, Garufi A, Vitabile S, Gilardi MC, Mauri G. Automated prostate gland segmentation based on an unsupervised fuzzy c-means clustering technique using multispectral $\mathrm{T} 1 \mathrm{w}$ and T2w MR imaging. Information, 2017. 8(2):49. doi:10.3390/info8020049.

[54] Luong NH, Alderliesten T, Bel A, Niatsetski Y, Bosman PA. Application and benchmarking of multiobjective evolutionary algorithms on high-dose-rate brachytherapy planning for prostate cancer treatment. Swarm Evol. Comput., 2018. 40:37-52. doi:10.1016/j.swevo.2017.12.003.

[55] Holland JH. Adaptation in Natural and Artificial Systems: An Introductory Analysis with Applications to Biology, Control and Artificial Intelligence. MIT Press, Cambridge, MA, USA, 1992. ISBN 0262082136.

[56] Rundo L, Tangherloni A, Nobile MS, Militello C, Besozzi D, Mauri G, Cazzaniga P. MedGA: a novel evolutionary method for image enhancement in medical imaging systems. Expert Syst. Appl., 2019. 119:387-399. doi:10.1016/j.eswa.2018.11.013.

[57] Hashemi S, Kiani S, Noroozi N, Moghaddam ME. An image contrast enhancement method based on genetic algorithm. Pattern Recognit. Lett., 2010. 31(13):1816-1824. doi:10.1016/j.patrec.2009.12.006. 
[58] Rundo L, Tangherloni A, Cazzaniga P, Nobile MS, Russo G, Gilardi MC, Vitabile S, Mauri G, Besozzi D, Militello C. A novel framework for MR image segmentation and quantification by using MedGA. Comput. Methods Programs Biomed., 2019. 176:159-172. doi:10.1016/j.cmpb.2019.04.016.

[59] Maulik U. Medical image segmentation using genetic algorithms. IEEE Trans. Inform. Technol. Biomed., 2009. 13(2):166-173. doi:10.1109/TITB.2008.2007301.

[60] Cagnoni S, Dobrzeniecki AB, Poli R, Yanch JC. Genetic algorithm-based interactive segmentation of 3D medical images. Image Vis. Comput., 1999. 17(12):881-895. doi:10.1016/S0262-8856(98)00166-8.

[61] Antonelli M, Cardoso MJ, Johnston EW, Appayya MB, Presles B, Modat M, Punwani S, Ourselin S. GAS: a genetic atlas selection strategy in multi-atlas segmentation framework. Med. Image Anal., 2019. 52:97-108. doi:10.1016/j.media.2018.11.007.

[62] Ortiz A, Górriz J, Ramírez J, Salas-Gonzalez D, Llamas-Elvira JM. Two fully-unsupervised methods for MR brain image segmentation using SOM-based strategies. Appl. Soft Comput., 2013. 13(5):2668-2682. doi:10.1016/j.asoc.2012.11.020.

[63] Aličković E, Subasi A. Breast cancer diagnosis using GA feature selection and rotation forest. Neural Comput. Applic., 2017. 28(4):753-763. doi:10.1007/s00521-015-2103-9.

[64] Koza JR. Genetic Programming: On the Programming of Computers by Means of Natural Selection. MIT Press, Cambridge, MA, USA, 1 edition, 1992. ISBN 0262111705.

[65] Bianco S, Ciocca G, Schettini R. Combination of video change detection algorithms by genetic programming. IEEE Trans. Evol. Comput., 2017. 21(6):914-928. doi:10.1109/TEVC.2017.2694160.

[66] Vanneschi L, Castelli M, Silva S. A survey of semantic methods in genetic programming. Genet. Program. Evolvable Mach., 2014. 15(2):195-214. doi:10.1007/s10710-013-9210-0.

[67] Castelli M, Trujillo L, Vanneschi L, Popovič A. Prediction of relative position of CT slices using a computational intelligence system. Appl. Soft Comput., 2016. 46:537-542. doi:10.1016/j.asoc.2015.09. 021.

[68] Poli R. Analysis of the publications on the applications of particle swarm optimisation. J. Artif. Evol. Appl., 2008. 2008:2-10. doi:10.1155/2008/685175.

[69] Storn R, Price K. Differential evolution-a simple and efficient heuristic for global optimization over continuous spaces. J. Glob. Optim., 1997. 11(4):341-359. doi:10.1023/A:1008202821328.

[70] Lei B, Tan EL, Chen S, Ni D, Wang T, Lei H. Reversible watermarking scheme for medical image based on differential evolution. Expert Syst. Appl., 2014. 41(7):3178-3188. doi:10.1016/j.eswa.2013.11.019.

[71] Eberhart RC, Shi Y, Kennedy J. Swarm Intelligence. Morgan Kaufmann, Burlington, MA, USA, 1 edition, 2001. ISBN 1558605959.

[72] Kennedy J, Eberhart R. Particle swarm optimization. In: Proc. International Conference on Neural Networks (ICNN), volume 4. IEEE, 1995 pp. 1942-1948. doi:10.1109/ICNN.1995.488968.

[73] Rundo L, Tangherloni A, Militello C, Gilardi MC, Mauri G. Multimodal medical image registration using particle swarm optimization: a review. In: Proc. Symposium Series on Computational Intelligence (SSCI). IEEE, 2016 pp. 1-8. doi:10.1109/SSCI.2016.7850261.

[74] Wachowiak MP, Smolíková R, Zheng Y, Zurada JM, Elmaghraby AS, et al. An approach to multimodal biomedical image registration utilizing particle swarm optimization. IEEE Trans. Evol. Comput., 2004. 8(3):289-301. doi:10.1109/TEVC.2004.826068. 
[75] Abdel-Basset M, Fakhry AE, El-Henawy I, Qiu T, Sangaiah AK. Feature and intensity based medical image registration using particle swarm optimization. J. Med. Syst., 2017. 41(12):197. doi:10.1007/ s10916-017-0846-9.

[76] Karaboga D, Basturk B. A powerful and efficient algorithm for numerical function optimization: artificial bee colony (ABC) algorithm. J. Glob. Optim., 2007. 39(3):459-471. doi:10.1007/s10898-007-9149-x.

[77] Zhang Y, Wu L. Optimal multi-level thresholding based on maximum Tsallis entropy via an artificial bee colony approach. Entropy, 2011. 13(4):841-859. doi:10.3390/e13040841.

[78] Dorigo M, Maniezzo V, Colorni A. Ant system: optimization by a colony of cooperating agents. IEEE Trans. Syst. Man Cybern., Part B, 1996. 26(1):29-41. doi:10.1109/3477.484436.

[79] Dorigo M, Stützle T. Ant colony optimization: overview and recent advances. In: Gendreau M, Potvin JY (eds.), Handbook of Metaheuristics, volume 272 of International Series in Operations Research \& Management Science, pp. 311-351. Springer, 2019. doi:10.1007/978-3-319-91086-4_10.

[80] Pereira C, Gonçalves L, Ferreira M. Optic disc detection in color fundus images using ant colony optimization. Med. Biol. Eng. Comput., 2013. 51(3):295-303. doi:10.1007/s11517-012-0994-5.

[81] Hassanien AE, Moftah HM, Azar AT, Shoman M. MRI breast cancer diagnosis hybrid approach using adaptive ant-based segmentation and multilayer perceptron neural networks classifier. Appl. Soft Comput., 2014. 14:62-71. doi:10.1016/j.asoc.2013.08.011.

[82] McCulloch WS, Pitts W. A logical calculus of the ideas immanent in nervous activity. Bull. Math. Biophys., 1943. 5(4):115-133. doi:10.1007/BF02478259.

[83] Kohonen T. Self-organized formation of topologically correct feature maps. Biol. Cybern., 1982. 43(1):59-69. doi:10.1007/BF00337288.

[84] LeCun Y, Bengio Y, Hinton G. Deep learning. Nature, 2015. 521(7553):436. doi:10.1038/nature145.

[85] Fukushima K. Neocognitron: a self-organizing neural network model for a mechanism of pattern recognition unaffected by shift in position. Biol. Cybern., 1980. 36(4):193-202. doi:10.1007/BF00344251.

[86] Hubel DH, Wiesel TN. Receptive fields, binocular interaction and functional architecture in the cat's visual cortex. J. Physiol., 1962. 160(1):106-154.

[87] Litjens G, Kooi T, Bejnordi BE, Setio AAA, Ciompi F, Ghafoorian M, et al. A survey on deep learning in medical image analysis. Med. Image Anal., 2017. 42:60-88. doi:10.1016/j.media.2017.07.005.

[88] Rajchl M, Lee MC, Oktay O, Kamnitsas K, Passerat-Palmbach J, Bai W, et al. DeepCut: object segmentation from bounding box annotations using convolutional neural networks. IEEE Trans. Med. Imaging, 2017. 36(2):674-683. doi:10.1109/TMI.2016.2621185.

[89] Havaei M, Davy A, Warde-Farley D, Biard A, Courville A, Bengio Y, et al. Brain tumor segmentation with deep neural networks. Med. Image Anal., 2017. 35:18-31. doi:10.1016/j.media.2016.05.004.

[90] Kamnitsas K, Ledig C, Newcombe VFJ, Simpson JP, Kane AD, Menon DK, et al. Efficient multi-scale 3D CNN with fully connected CRF for accurate brain lesion segmentation. Med. Image Anal., 2017. 36:61-78. doi:10.1016/j.media.2016.10.004.

[91] Ronneberger O, Fischer P, Brox T. U-Net: convolutional Networks for biomedical image segmentation. In: Proc. Conference on Medical Image Computing and Computer-Assisted Intervention (MICCAI), volume 9351 of $L N C S$. Springer, 2015 pp. 234-241. doi:10.1007/978-3-319-24574-4_28. 
[92] Milletari F, Navab N, Ahmadi SA. V-Net: fully convolutional neural networks for volumetric medical image segmentation. In: Proc. International Conference on 3D Vision (3DV). IEEE, 2016 pp. 565-571. doi:10.1109/3DV.2016.79.

[93] Schlemper J, Oktay O, Schaap M, Heinrich M, Kainz B, Glocker B, Rueckert D. Attention gated networks: learning to leverage salient regions in medical images. Med. Image Anal., 2019. 53:197-207. doi:10.1016/j.media.2019.01.012.

[94] Pelt DM, Sethian JA. A mixed-scale dense convolutional neural network for image analysis. Proc. Natl. Acad. Sci., 2018. 115(2):254-259. doi:10.1073/pnas.1715832114.

[95] Goodfellow I, Pouget-Abadie J, Mirza M, Xu B, Warde-Farley D, Ozair S, et al. Generative adversarial nets. In: Proc. Advances in Neural Information Processing Systems (NIPS). 2014 pp. 2672-2680. http://papers.nips.cc/paper/5423-generative-adversarial-nets.

[96] Zhu JY, Park T, Isola P, Efros AA. Unpaired image-to-image translation using cycle-consistent adversarial networks. In: Proc. International Conference on Computer Vision (ICCV). IEEE, 2017 pp. 2242-2251. doi:10.1109/ICCV.2017.244.

[97] Gulrajani I, Ahmed F, Arjovsky M, Dumoulin V, Courville AC. Improved training of Wasserstein GANs. In: Advances in Neural Information Processing Systems. 2017 pp. 5769-5779.

[98] Ravì D, Wong C, Deligianni F, et al. Deep learning for health informatics. IEEE J. Biomed. Health Inform., 2017. 21(1):4-21. doi:10.1109/JBHI.2016.2636665.

[99] Costa P, Galdran A, Meyer MI, Niemeijer M, Abràmoff M, Mendona AM, Campilho A. End-to-end adversarial retinal image synthesis. IEEE Trans. Med. Imaging, 2018. 37(3):781-791. doi:10.1109/TMI. 2017.2759102.

[100] Chuquicusma MJM, Hussein S, Burt J, Bagci U. How to fool radiologists with generative adversarial networks? A visual Turing test for lung cancer diagnosis. arXiv preprint arXiv:1710.09762v1, 2017.

[101] Frid-Adar M, Diamant I, Klang E, Amitai M, Goldberger J, Greenspan H. GAN-based synthetic medical image augmentation for increased CNN performance in liver lesion classification. Neurocomputing, 2018. 321:321-331. doi:10.1016/j.neucom.2018.09.013.

[102] Han C, Hayashi H, Rundo L, Araki R, Shimoda W, Muramatsu S, et al. GAN-based synthetic brain MR image generation. In: Proc. International Symposium on Biomedical Imaging (ISBI). IEEE, 2018 pp. 734-738. doi:10.1109/ISBI.2018.8363678.

[103] Prastawa M, Bullitt E, Gerig G. Simulation of brain tumors in MR images for evaluation of segmentation efficacy. Med. Image Anal., 2009. 13(2):297-311. doi:10.1016/j.media.2008.11.002.

[104] Yao X. Evolving artificial neural networks. Proc. IEEE, 1999. 87(9):1423-1447. doi:10.1109/5.784219.

[105] Stanley KO, D'Ambrosio DB, Gauci J. A hypercube-based encoding for evolving large-scale neural networks. Artificial Life, 2009. 15(2):185-212. doi:10.1162/artl.2009.15.2.15202.

[106] Stanley KO, Miikkulainen R. Evolving neural networks through augmenting topologies. Evol. Comput., 2002. 10(2):99-127. doi:10.1162/106365602320169811.

[107] Calimeri F, Marzullo A, Stamile C, Terracina G. Blood vessel segmentation in retinal fundus images using Hypercube NeuroEvolution of augmenting topologies (HyperNEAT). In: Quantifying and Processing Biomedical and Behavioral Signals, volume 103 of Smart Innovation, Systems and Technologies, pp. 173-183. Springer, 2019. doi:10.1007/978-3-319-95095-2_17. 
[108] Gatenby RA, Grove O, Gillies RJ. Quantitative imaging in cancer evolution and ecology. Radiology, 2013. 269(1):8-14. doi:10.1148/radiol.13122697.

[109] Evanko D. Two pictures are better than one. Nat. Methods, 2008. 5(5):377. doi:10.1038/nmeth0508-377.

[110] Gillies RJ, Kinahan PE, Hricak H. Radiomics: images are more than pictures, they are data. Radiology, 2015. 278(2):563-577. doi:10.1148/radiol.2015151169.

[111] Lambin P, Rios-Velazquez E, Leijenaar R, Carvalho S, van Stiphout RG, Granton P, Zegers CM, Gillies R, Boellard R, Dekker A, et al. Radiomics: extracting more information from medical images using advanced feature analysis. Eur. J. Cancer, 2012. 48(4):441-446. doi:10.1016/j.ejca.2011.11.036.

[112] Lambin P, Leijenaar RT, Deist TM, Peerlings J, de Jong EE, van Timmeren J, Sanduleanu S, Larue RT, Even AJ, Jochems A, et al. Radiomics: the bridge between medical imaging and personalized medicine. Nat. Rev. Clin. Oncol., 2017. 14(12):749. doi:10.1038/nrclinonc.2017.141.

[113] Rosenstein BS, West CM, Bentzen SM, Alsner J, Andreassen CN, Azria D, Barnett GC, Baumann M, Burnet N, Chang-Claude J, et al. Radiogenomics: radiobiology enters the era of big data and team science. Int. J. Radiat. Oncol. Biol. Phys., 2014. 89(4):709-713. doi:10.1016/j.ijrobp.2014.03.009.

[114] Sala E, Mema E, Himoto Y, Veeraraghavan H, Brenton J, Snyder A, Weigelt B, Vargas HA. Unravelling tumour heterogeneity using next-generation imaging: radiomics, radiogenomics, and habitat imaging. Clin. Radiol., 2017. 72(1):3-10. doi:10.1016/j.crad.2016.09.013.

[115] Brindle K. New approaches for imaging tumour responses to treatment. Nat. Rev. Cancer, 2008. 8(2):94. doi:10.1038/nrc2289.

[116] Yuan Y, Failmezger H, Rueda OM, Ali HR, Gräf S, Chin SF, Schwarz RF, Curtis C, Dunning MJ, Bardwell $\mathrm{H}$, et al. Quantitative image analysis of cellular heterogeneity in breast tumors complements genomic profiling. Sci. Transl. Med., 2012. 4(157):157ra143-157ra143. doi:10.1126/scitranslmed.3004330.

[117] Tangherloni A, Spolaor S, Rundo L, Nobile MS, Cazzaniga P, Mauri G, Liò P, Merelli I, Besozzi D. GenHap: a novel computational method based on genetic algorithms for haplotype assembly. BMC Bioinform., 2019. 20(Suppl 4):172. doi:10.1186/s12859-019-2691-y.

[118] Hassanien AE, Abraham A, Peters JF, Schaefer G, Henry C. Rough sets and near sets in medical imaging: a review. IEEE Trans. Inf. Technol. Biomed., 2009. 13(6):955-968. doi:10.1109/TITB.2009.2017017.

[119] Zadeh LA. Fuzzy logic = computing with words. IEEE Trans. Fuzzy Syst., 1996. 4(2):103-111. doi: 10.1109/91.493904.

[120] Militello C, Vitabile S, Rundo L, Russo G, Midiri M, Gilardi MC. A fully automatic 2D segmentation method for uterine fibroid in MRgFUS treatment evaluation. Comput. Biol. Med., 2015. 62:277-292. doi:10.1016/j.compbiomed.2015.04.030.

[121] Rundo L, Militello C, Tangherloni A, Russo G, Vitabile S, Gilardi MC, Mauri G. NeXt for neuroradiosurgery: a fully automatic approach for necrosis extraction in brain tumor MRI using an unsupervised machine learning technique. Int. J. Imaging Syst. Technol., 2018. 28(1):21-37. doi: 10.1002/ima.22253.

[122] Nobile MS, Cazzaniga P, Besozzi D, Colombo R, Mauri G, Pasi G. Fuzzy Self-Tuning PSO: a settingsfree algorithm for global optimization. Swarm Evol. Comput., 2018. 39:70-85. doi:10.1016/j.swevo. 2017.09.001. 
[123] Tangherloni A, Rundo L, Nobile MS. Proactive particles in swarm optimization: a settings-free algorithm for real-parameter single objective optimization problems. In: Proc. IEEE Congress on Evolutionary Computation (CEC). IEEE, 2017 pp. 1940-1947. doi:10.1109/CEC.2017.7969538.

[124] Nobile MS, Tangherloni A, Rundo L, Spolaor S, Besozzi D, Mauri G, Cazzaniga P. Computational intelligence for parameter estimation of biochemical systems. In: Proc. Congress on Evolutionary Computation (CEC). IEEE, 2018 pp. 1-8. doi:10.1109/CEC.2018.8477873.

[125] Mastriani M. Quantum image processing? Quantum Inf. Process., 2017. 16(1):27. doi:10.1007/ s11128-016-1457-y.

[126] Yan F, Iliyasu AM, Jiang Z. Quantum computation-based image representation, processing operations and their applications. Entropy, 2014. 16(10):5290-5338. doi:10.3390/e16105290.

[127] Tangherloni A, Rundo L, Spolaor S, Nobile M, Merelli I, Besozzi D, Mauri G, Cazzaniga P, Liò P. High performance computing for haplotyping: models and platforms. In: Mencagli G, et al. (eds.), Proc. 24th International European Conference on Parallel and Distributed Computing (Euro-Par 2018), Workshop on Advances in High-Performance Bioinformatics, Systems Biology (Med-HPC 2018), volume 11339 of LNCS. Springer, 2019 pp. 650-661. doi:10.1007/978-3-030-10549-5\_51.

[128] Nobile MS, Cazzaniga P, Tangherloni A, Besozzi D. Graphics processing units in bioinformatics, computational biology and systems biology. Brief. Bioinform., 2016. 18(5):870-885. doi:10.1093/bib/bbw058.

[129] Eklund A, Dufort P, Forsberg D, LaConte SM. Medical image processing on the GPU-past, present and future. Med. Image Anal., 2013. 17(8):1073-1094. doi:10.1016/j.media.2013.05.008.

[130] Smistad E, Falch TL, Bozorgi M, Elster AC, Lindseth F. Medical image segmentation on GPUs-a comprehensive review. Med. Image Anal., 2015. 20(1):1-18. doi:10.1016/j.media.2014.10.012.

[131] Tajbakhsh N, Shin JY, Gurudu SR, et al. Convolutional neural networks for medical image analysis: full training or fine tuning? IEEE Trans. Med. Imaging, 2016. 35(5):1299-1312. doi:10.1109/TMI.2016. 2535302.

[132] Criminisi A. Machine learning for medical images analysis. Med. Image Anal., 2016. 33:91-93. doi: 10.1016/j.media.2016.06.002.

[133] Cabitza F, Rasoini R, Gensini GF. Unintended consequences of machine learning in medicine. J. Am. Med. Assoc., 2017. 318(6):517-518. doi:10.1001/jama.2017.7797.

[134] Gambino O, Rundo L, Cannella V, Vitabile S, Pirrone R. A framework for data-driven adaptive GUI generation based on DICOM. J. Biomed. Inform., 2018. 88:37-52. doi:10.1016/j.jbi.2018.10.009. 